\title{
DOI: https://doi.org/10.46296/rc.v4i8edesp.0036
}

\section{San Lorenzo: Zona vulnerable a la narco guerrilla \\ San Lorenzo: Zone vulnerable to the narco guerrilla}

\author{
Chávez-Vaca Vinicio Alexander \\ Docente investigador de la Carrera de Medicina Veterinaria, Escuela Superior Politécnica \\ Agropecuaria de Manabí "Manuel Félix López" ESPAM MFL. Calceta, Ecuador. \\ ORCID ID: https://orcid.org/0000-0003-3623-4178 \\ Correo: vachavez@espam.edu.ec

\section{González-Zambrano Rosa} \\ Doctora en Ciencias Pedagógicas, Docente investigadora de la Carrera de Medicina \\ Veterinaria, Escuela Superior Politécnica Agropecuaria de Manabí "Manuel Félix López" \\ ESPAM MFL. Calceta, Ecuador. \\ ORCID ID: https://orcid.org/0000-0003-0960-1817 \\ Correo: rgonzales@espam.edu.ec
}

\begin{abstract}
RESUMEN
El presente estudio se propuso determinar los factores geográficos, sociales, económicos y de seguridad ciudadana que enlazan al cantón San Lorenzo, perteneciente a la provincia de Esmeraldas en Ecuador, con bandas narcotraficantes colombianas, así como las consecuencias de convertirse en zona de guerra, a raíz del conflicto fronterizo. El procedimiento metodológico fue de enfoque cualitativo y se empleó como principal técnica el análisis de documento, con lo cual fue posible concluir que las consecuencias de la influencia, presencia y operatividad de los grupos subversivos y del crimen organizado en este territorio ecuatoriano generan ondas para todo el país y evidencian cómo el auge de estos conflictos no solo se relaciona con las operaciones de los grupos guerrilleros, sino también con el desarrollo y las acciones gubernamentales a favor de la ciudadanía y el bienestar de sus pueblos.
\end{abstract}

Palabras claves: seguridad ciudadana, cantón San Lorenzo, narcotráfico, crimen organizado, conflicto fronterizo.

\begin{abstract}
The present study aimed to determine the geographic, social, economic and citizen security factors that link the San Lorenzo canton, belonging to the province of Esmeraldas in Ecuador, with Colombian drug gangs, as well as the consequences of becoming a war zone in the wake of the border conflict. The methodological procedure was a qualitative approach and the document analysis was used as the main technique, which made it possible to conclude that the consequences of the influence, presence and operation of subversive groups and organized crime in this Ecuadorian territory generate waves for all the country and show how the rise of these conflicts is not only related to the operations of the guerrilla groups, but also to the development and governmental actions in favor of citizenship and the welfare of their peoples.
\end{abstract}

Keywords: citizen security, San Lorenzo canton, drug trafficking, organized crime, border conflict.

Información del manuscrito:

Fecha de recepción: 06 de septiembre de 2021.

Fecha de aceptación: 08 de noviembre de 2021.

Fecha de publicación: 09 de noviembre de 2021. 


\section{INTRODUCCIÓN}

En medio del aún vigente proceso de paz en Colombia, las tensiones en su frontera con Ecuador se intensifican luego del primer trimestre de 2018. Asaltos con coches de bomba, declaración de estado de excepción, pérdida de vidas humanas, secuestros, heridos y despliegue de la fuerza militar caracterizan este conflicto que ha tomado notoriedad en los últimos meses, pero con causas añejas que no solo se relacionan con el narcotráfico.

Las condiciones socioeconómicas y el impacto de las políticas públicas en el cantón de San Lorenzo aportan elementos para entender el detonante de un problema anunciado. Diez años de conversaciones entre Ecuador y Colombia sobre la paz no es, ni ha sido suficiente para evitar la ya muy cerca guerra de guerrilla en escenario ecuatoriano, a partir de la lucha de los grupos armados para continuar con el narcotráfico, el contrabando y la explotación ilegal de recursos.

Es por ello que con este artículo se pretenden determinar los factores geográficos, sociales, económicos y de seguridad ciudadana que enlazan al cantón San Lorenzo con los narcos, así como la consecuencia de ser partícipe del crimen organizado. Para dar respuesta a este interés se emplea un enfoque metodológico cualitativo, destacando fundamentalmente el análisis de documento. De ahí que la revisión de la bibliografía existente sobre el tema es la base de toda la información que se expone seguidamente sobre el cantón San Lorenzo, su realidad y las consecuencias de su relación directa o indirecta con exguerrilleros colombianos.

\section{DESARROLLO}

\subsection{Contextualizando al cantón San Lorenzo}

San Lorenzo es uno de los 221 cantones que existen en Ecuador. Forma parte de la división administrativa de segundo nivel y actualmente pertenece a la provincia de Esmeraldas. Con anterioridad, el cantón formaba parte de la provincia de Imbabura, pero la ausencia de conexión vial provocó que se devolviera a la actual provincia. 
En la historia del cantón destacan otros detalles en relación a su pertenencia. Por ejemplo, en la década sesenta del siglo XIX, sus territorios se canjearon por la deuda del país con los ingleses, por lo que se cedieron a la compañía "Ecuador Land". Así surgió una especie de territorio independiente del ordenamiento jurídico nacional, estableciéndose, incluso, una moneda propia (pailón). Hasta los primeros 30 años del siglo XX se mantuvo esta situación y a partir de ese año, San Lorenzo fue devuelto a Ecuador (Chuquizala \& Jaramillo, 2017).

Desde enero de 2018, ocupa titulares en los medios de comunicación nacionales e internacionales. Se ha convertido en zona de guerra por el conflicto con exmiembros de la guerrilla colombiana, lo cual se relaciona con su situación geográfica. San Lorenzo comparte límites fronterizos con la República de Colombia (norte), además de su conexión con el cantón Eloy Alfaro (sur) también limita con Carchi, otra provincia ecuatoriana (oeste), y con el Océano Pacífico (oeste).

Y es precisamente esa cercanía con el otro país, lo que genera que sea parte del crimen organizado, pues en esta área del territorio colombiano (principalmente Nariño) se cultiva la mayor cantidad de hoja de coca y están las fábricas donde la planta se transforma en pasta base. De acuerdo con la Oficina de Naciones Unidas contra la Droga y el delito (UNODC, 2017), el cultivo de coca pasó de 96.000ha en 2015 a 146.000ha en 2016, principalmente aumentó en Nariño, no obstante, en esta zona como parte del proceso de paz no se ha intensificado la sustitución o erradicación manual de las plantaciones, existen cerca de 10 000ha de sembradíos ilícitos. Además, en la situación de San Lorenzo también influye que el Océano Pacífico es el "principal escenario del tráfico de drogas desde Colombia hacia México, en cuyo trayecto se involucra a Ecuador" (Cadena, 2010, p. 46).

A la amenaza que representa esta situación para el mantenimiento del crimen organizado se agregan otros factores que hacen de San Lorenzo, zona vulnerable ante el narco. Este cantón, según datos del Instituto Nacional de Estadística y Censos (INEC, 2010), tiene una población de 42.486, de la cual más del $30 \%$ no es originaria y se encuentra distribuida de la siguiente forma (Tabla 1): 
Tabla 1. Distribución poblacional por área.

\begin{tabular}{ll}
\hline Población por área & Cantidad \\
\hline Área urbana & 23.265 \\
Área rural & 19.221 \\
Total & $\mathbf{4 2 . 4 8 6}$ \\
\hline
\end{tabular}

Fuente: INEC (2010).

De acuerdo con Moncayo (2012), transformaciones demográficas, territoriales y socioculturales constituyen procesos característicos de este cantón en los últimos años. En general, ha ocurrido un acelerado incremento de la población, han desaparecido territorios ancestrales y aunque han mejorado los indicadores de seguridad ciudadana, la violencia y las actividades ilegales continúan amenazando el buen vivir. A ello se suma la existencia de una realidad de desarrollo social y económica compleja, a pesar de las políticas públicas, las perspectivas de desarrollo integral no han sido muy incidentes en esta parte del territorio ecuatoriano.

\subsection{Oportunidades para el desarrollo en San Lorenzo: ¿presentes o difusas?}

¿Solo la cercanía geográfica provoca que San Lorenzo constituya un cantón relacionado con los actores del crimen organizado? Si se buscan respuestas a esta interrogante se identifica que otros factores sostienen y viabilizan esa situación. A criterio de Bagley (2015), el accionar de los grupos narcotraficantes y la complicidad de algunos ciudadanos es más incidente en aquellos territorios que facilitan algunos de los procesos de esta actividad ilícita, pero también donde las políticas públicas no han sido muy influyentes y centradas a disminuir los riesgos, lo cual tiene aplicabilidad en el territorio ecuatoriano que se toma como unidad de análisis para este artículo.

Al indagar sobre algunos de los parámetros para medir la pobreza por necesidades básicas insatisfechas (NBI) se conoce que en San Lorenzo persisten algunas problemáticas, como se muestra en la tabla 2 : 
Tabla 2. Cobertura de servicios en viviendas.

\begin{tabular}{ccc}
\hline Cobertura de servicios & Provincial & Cantonal \\
\hline $\begin{array}{c}\text { Servicio de energía eléctrica } \\
\text { Eliminación de aguas servidas } \\
\text { por red pública }\end{array}$ & 97,3 & 79,43 \\
$\begin{array}{c}\text { Abastecimiento de agua por } \\
\quad \text { red pública }\end{array}$ & 64,1 & 27,32 \\
$\begin{array}{c}\text { Eliminación de basura por } \\
\text { carro recolector }\end{array}$ & 55,5 & 64,08 \\
\hline
\end{tabular}

Fuente: INEC (2010).

De lo anterior puede reconocerse que existen diferencias entre la cobertura de la provincia y el cantón. Las mayores dificultades se reportan con el abastecimiento de agua. No obstante, el comportamiento de esos servicios permite plantear que las condiciones de vida no son las adecuadas para algunos pobladores.

La pesca artesanal, las actividades madereras y el comercio constituyen las principales fuentes de ingreso de este territorio ecuatoriano. De ahí que el discreto desarrollo económico sea otra de las características presentes en San Lorenzo que atentan contra la calidad de vida de la población, incidiendo de manera significativa en el mercado laboral.

A pesar de ello, en San Lorenzo la cobertura de otros servicios públicos y privados sí está presente. Por ejemplo, se cuenta con instituciones educativas, instituciones de salud y el sistema de protección a la seguridad ciudadana. No obstante, a raíz de los sucesos de enero de 2018, los pobladores han reconocido que con anterioridad, la presencia de los miembros del orden público no era tan latente como luego de las actividades criminales y la consolidación del conflicto.

Solo existían cerca de 85 policías para realizar el control urbano, lo cual a criterio del alcalde de San Lorenzo, Gustavo Samaniego, es una debilidad (El Telégrafo, 2018). Además, debido al comportamiento de la acción gubernamental, el catón sigue requiriendo de políticas enfocadas a la revitalización de espacios para garantizar el bienestar ciudadano, no solo se exige mayor igualdad de oportunidades, sino también proyectos para mejorar el espacio público y 
promover el emprendimiento, con lo cual surgen nuevas fuentes de ingreso y se propicia que algunos pobladores no vean al narcotráfico como la solución a sus problemas y a sus carencias.

En la Ley de Seguridad Pública y del Estado se establece que "la seguridad ciudadana es una política de Estado, destinada a fortalecer y modernizar los mecanismos necesarios para garantizar el derecho a una vida libre de violencia y criminalidad" (Art. 23, Ley de Seguridad Pública y del Estado, 2009). "La política del gobierno es luchar contra la delincuencia organizada" (Observatorio Metropolitano de Seguridad Ciudadana, OMSC, 2013, p. 25). No obstante, en el territorio esmeraldeño que se toma como objeto de estudio, el delito continúa debido a la debilidad de protección en las fronteras, la fallas en el funcionamiento de instituciones públicas y agentes claves como la Policía Nacional, así como la complicidad de la población, ya sea por miedo o por la recepción de beneficios como parte del desarrollo de las actividades ilegales.

Lo anterior puede verse como una consecuencia del deprimido mercado laboral del cantón. Es generalizada y ha sido constante la ausencia de ofertas de empleo, lo cual ha provocado que algunos habitantes, principalmente los más jóvenes, se relacionen con los narcos. Para Moncayo (2012), por lo general, los que toman esas decisiones provienen de familias en condiciones de pobreza y disfuncionales, por lo cual ven en las actividades ilegales una oportunidad de cambiar su vida, pues como se conoce el negocio de la droga es arriesgado, pero muy lucrativo. Por ejemplo, se ha demostrado que el tráfico es muy bien remunerado por el valor que tiene la droga en otros mercados; en Colombia un kilo de cocaína puede alcanzar los 4 millones de pesos, pero en China puede subir 496 millón más, es decir, cerca de USD\$170.000 (El Tiempo, 2017).

Por otro lado, no puede dejar de reconocerse que, en San Lorenzo, la incautación de droga aumentó de 2016 a 2017, pero, el número de casos y los detenidos disminuyeron (Tabla 3), lo cual puede conllevar a dos interpretaciones: concentración de mercancía en menos involucrados y poca efectividad en las acciones policiales, provocando fuga de los participantes o no identificación de la actividad ilegal, lo cual en otras palabras podría resultar en inobservancia al crimen organizado. 
Tabla 3. Incautación de droga en San Lorenzo.

\begin{tabular}{cccc}
\hline Cifras & $\mathbf{2 0 1 6}$ & AÑOS & $\mathbf{2 0 1 8}$ \\
\hline Incautaciones & $772.408,50$ & $\mathbf{2 0 1 7}$ & $6.860,00$ \\
Casos & 76 & 61 & 5 \\
Detenidos & 98 & 68 & 10 \\
\hline
\end{tabular}

Fuente: Policía Judicial (2018).

En cuanto a los delitos en el cantón San Lorenzo se identifica una disminución de estas acciones de manera general, lo cual estaría relacionado con una discreta mejora de la seguridad ciudadana, pero solo en términos relativos, pues los decrecimientos no son significativos, incluso, algunas actividades aumentaron como muestra la figura 1:

Figura 1. Comportamiento del delito en el cantón San Lorenzo.

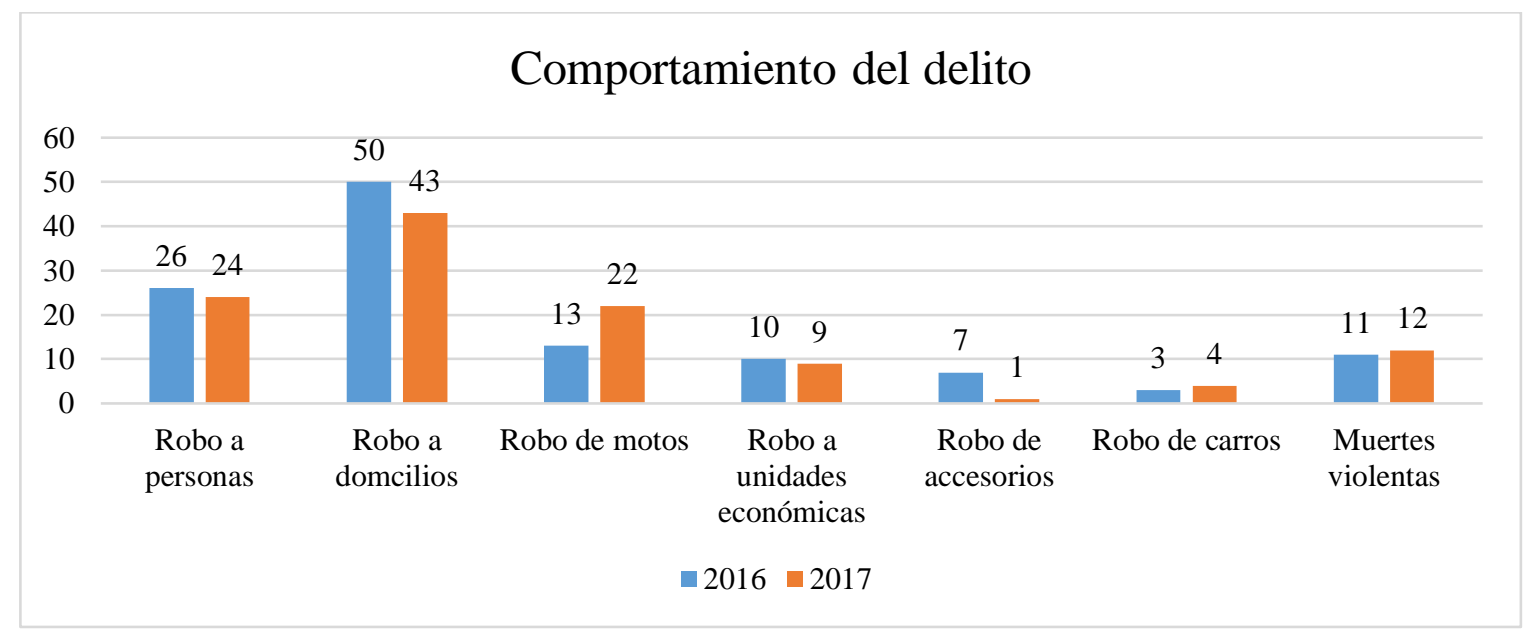

Fuente: Policía Judicial (2018)

A pesar del comportamiento de los delitos y de las actividades de incautación, la información más actualizada sobre el cantón permite identificar contradicciones. Si bien se reporta una reducción de los índices de inseguridad, este territorio es escenario del accionar de las bandas transnacionales del tráfico de droga.

Todas estas condiciones, las particularidades socioeconómicas, la ubicación geográfica y el poco incentivo al desarrollo hacen del cantón San Lorenzo un escenario vulnerable del crimen organizado, lo cual provoca consecuencias para sus pobladores y para el interés nacional de garantizar la seguridad ciudadana. 


\subsection{Influencia del crimen organizado y sus consecuencias}

Las investigaciones y operativos emanados del conflicto fronterizo iniciado en 2018, permitieron a las autoridades ecuatorianas de inteligencia identificar que al cantón San Lorenzo llegan los impactos de las operaciones de seis grupos delictivos, asentados en Nariño. El anhelado control de las fronteras, que se planteaba desde el acuerdo de paz, no ha sido evidente y demuestra las distorsiones en este proceso, afectando con ello a naciones cercanas como Ecuador.

El Clan del Golfo, el Frente Oliver Sinisterra (el de mayor acción militar en la zona que se estudia), las Guerrillas Unidas del Pacífico (GUP), Resistencia Campesina, Gente de Orden y La Empresa (El Telégrafo, 2018) son las bandas que en los últimos meses han afectado, aún más, la seguridad de territorios fronterizos del país, causando con ello pérdidas de vidas humanas, temor, secuestros, conflictos y mayores gastos en el despliegue de las fuerzas militares. Esto evidencia lo planteado por Guerrón (2000) al señalar que el narcotráfico ocasiona disímiles implicaciones socioeconómicas y políticas, lo cual provoca que se dificulte su tratamiento, porque, además, hay muchos intereses en juego y destacan percepciones de todo tipo sobre el problema.

Una muestra de ello, es el posible recrudecimiento del conflicto en un futuro, debido a problemas internos en los propios grupos criminales. De acuerdo a la Fundación Paz y Reconciliación, existe una asociación muy débil entre el Clan del Golfo y las GUP, lo cual puede terminar en un enfrentamiento armado entre estas dos agrupaciones de exguerrilleros por el poder y el control de la zona colombiana de Tumaco, lo cual pudiera generar mayores inseguridades en la frontera, abarcando el territorio ecuatoriano (El Comercio, 2018).

Para Cadena (2010), existen nuevos actores en el contexto nacional e internacional que primeramente impactan localmente y luego esas consecuencias se generalizan al ámbito nacional e incluso externo. Ello obliga a las autoridades a transformar la dinámica de diseñar e implementar políticas, con la finalidad de que el ordenamiento jurídico y las acciones gubernamentales den respuesta y atención a las modalidades criminales. 
Además, la operación de estos grupos conformados por exguerrilleros corrobora, una vez más, que en el tema de la droga Ecuador desempeña el rol de país de tránsito. Por aquí pasa la droga que llega a otros mercados, principalmente a los del norte del continente americano. Si se emplean otros términos, se plantea que el cantón es uno de los nodos que interconecta a los narcos colombianos con el resto de los integrantes de estos grupos en el mundo y en el continente. Para Barragán (2015), los límites marítimos propician que por la parte oeste de San Lorenzo lleguen los productos complementarios para obtener el producto final y que desde aquí se distribuyan los ya procesados.

Actualmente, en el cantón San Lorenzo se desarrollan operaciones ilegales relacionadas también con el contrabando de combustible y el tráfico de armas, explosivos y personas. Además, se ha recurrido al secuestro, culminando en la muerte de tres civiles (hasta finales de marzo de 2018 fueron profesionales de la prensa ecuatoriana). Sin embargo, este accionar no parece ser una novedad en San Lorenzo. Desde 2012, Moncayo (2012) identificó que una modalidad de trata en el cantón era el reclutamiento forzoso y voluntario, mediante los cuales se suman personas a las filas de los grupos narcotraficantes ya sea para combatir, trabajar en las plantaciones o comercializar la droga.

Por lo tanto, la realidad del cantón San Lorenzo se torna difícil y compleja para mantener la seguridad ciudadana y el buen vivir de la población. Se reportan desplazamientos de familias de Mataje, una de las parroquias del cantón (Cordero, 2018), de intensificarse más el conflicto podrían desaparecer asentamientos rurales, por el temor de la población. Ante esas posibles consecuencias y tras el intento de no desproteger a los ciudadanos, desde el Ministerio de Educación en el país, se ha trazado una estrategia para las instituciones educativas que incluye tres etapas de acompañamiento y prevención en temas psicoemocionales, así como programas de educación para la paz.

Mientras se espera por los buenos resultados, las consecuencias de la influencia, presencia y operatividad de los grupos subversivos y del crimen organizado en este territorio ecuatoriano generan ondas para todo el país y evidencian cómo el auge de estos conflictos puede relacionarse con el discreto desarrollo y las pocas 
acciones gubernamentales a favor de la ciudadanía y el bienestar de sus pueblos. El tipo de final de la situación fronteriza que inició en enero de 2018 todavía no se vislumbra, lo cual no impide que se reconozcan, además de la cercanía a la actividad ilícita de los narcos, otras causas del conflicto.

\section{CONCLUSIONES}

Luego de la exposición realizada con anterioridad, que resultó del análisis de documento, puede concluirse que:

La situación geográfica del cantón San Lorenzo, sus límites con el Océano Pacífico y con territorios colombianos, con grandes plantaciones de coca, donde operan exguerrilleros no acogidos o inconformes con el proceso de paz, provoca que este territorio ecuatoriano sea escenario de la acción del crimen organizado y centro del conflicto fronterizo que inició en enero de 2018 y que convirtió al territorio en zona de guerra.

Las particularidades socioeconómicas como el deprimido mercado laboral, la pobreza, los problemas con la satisfacción de necesidades básicas, el estado de los espacios públicos y las debilidades en la garantía de la seguridad ciudadana hacen del cantón San Lorenzo, un escenario vulnerable del crimen organizado, lo cual provoca consecuencias para sus pobladores y para todo el Ecuador.

Las operaciones de seis grupos de exguerrilleros colombianos afectan, aún más, la seguridad y el normal transcurrir de la vida en el cantón fronterizo de San Lorenzo, causando con ello pérdidas de vidas humanas, temor, secuestros, conflictos, amenazas a la seguridad, ejemplos de trata y mayores gastos en el despliegue de las fuerzas militares ecuatorianas.

\section{REFERENCIAS}

Bagley, B. (2015). Ecuador: ¿país de tránsito de cocaína? Perfil Criminológico, 13.

Barragán, J. C. (2015). Política pública sostenida para preservar a la ciudadanía. Perfil Criminológico, 10-12. 
Cadena, J. L. (2010). Geopolítica del narcotráfico. México y Colombia: la equivocación en el empleo de las fuerzas militares. Bogotá: Universidad de Santo Tomas.

Chuquizala, T., \& Jaramillo, B. (2017). Plan de promoción turística para el cantón San Lorenzo del Pailón, provincia de Esmeraldas. Siembra, 4(1), 121130.

Cordero, B. (2018). Situación en San Lorenzo. Quito: Ministerio de Inclusión Económica y Socia.

El Comercio. (25 de febrero de 2018). Tres bandas operan frente a San Lorenzo. Recuperado de http://www.elcomercio.com/actualidad/sanlorenzobandas-operan-colombia-frontera.html.

El Telégrafo. (3 de febrero de 2018). San Lorenzo enfrenta el crimen organizado. Recuperado de https://www.eltelegrafo.com.ec/noticias/ecuador/1/sanlorenzo-enfrenta-el-crimen-organizado

El Tiempo. (5 de marzo de 2017). Un gramo de cocaína vale 500 dólares en Pekín. Recuperado de http://www.eltiempo.com/justicia/un-gramo-decocaina-colombiana-vale-500-dolares-en-pekin-64254

Guerrón, H. (2000). El narcotráfico y la subversión colombiana y su influencia en la región. Quito: Instituto de Altos Estudios Nacionales .

INEC. (2010). Censo de población y vivienda. Quito: INEC.

Moncayo, M. (2012). Diagnóstico sobre la trata de personas en el cantón San Lorenzo, provincia de Esmeralda. Quito: FLACSO-OIM.

OMSC. (2013). Estudios de seguridad ciudadana. Quito: OMSC.

Policía Judicial. (2018). Datos de San Lorenzo. Quito: Policía Judicial.

UNODC. (2017). Colombia: Monitoreo de territorios afectados por cultivos ilícitos 2016. Viena: UNODC. 